\title{
Supporting information Use of Ion Exchange To Regulate the Heterogeneous Ice Nucleation Efficiency of Mica
}

Shenglin Jin $\$ a$, Yuan Liu ${ }^{\ddagger b, d}$, Malte Deiseroth ${ }^{\ddagger c}$, Jie Liu ${ }^{\dagger a}$, Ellen H.G. Backus ${ }^{c, f}$, Hui Li $^{b}$, Han Xue $^{a}$, Lishan Zhao ${ }^{a}$, Xiao Cheng Zeng*d, Mischa Bonn*c, Jianjun Wang*a,e.

${ }^{a}$ Key Laboratory of Green Printing, Beijing National Laboratory for Molecular Science, Institute of Chemistry, Chinese Academy of Sciences, Beijing 100190, China.

${ }^{b}$ Beijing Advanced Innovation Center for Soft Matter Science and Engineering, Beijing University of Chemical Technology, Beijing 100029, China.

${ }^{c}$ Max Planck Institute for Polymer Research, 55128 Mainz, Germany.

${ }^{d}$ Department of Chemistry, University of Nebraska-Lincoln, Lincoln, Nebraska 68588, United States.

${ }^{e}$ School of Chemistry and Chemical Engineering, University of Chinese Academy of Sciences, Beijing 100049, China.

${ }^{f}$ Department of Physical Chemistry, University of Vienna, Wahringer Strasse 42, 1090 Wien, Austria.

\section{Corresponding Authors}

*xzeng1@unl.edu

* bonn@mpip-mainz.mpg.de

*wangj220@iccas.ac.cn

† These authors contributed equally.

Present Addresses

${ }^{\dagger}$ J. L.: Max Planck Institute for Polymer Research, 55128 Mainz, Germany. 


\section{Experimental Studies}

General. Sodium chloride $(\mathrm{NaCl})$, potassium chloride $(\mathrm{KCl})$, rubidium chloride $(\mathrm{RbCl})$ and cesium chloride $(\mathrm{CsCl})$ were purchased from Sigma Aldrich. Hydrochloric acid $37 \%(\mathrm{HCl})$ was purchased from Beijing Chemical Works and Sigma Aldrich. Microscope cover glasses $(24 \times 24 \mathrm{~mm})$ were prepared using a previously reported method $^{1}$ and purchased from CITOGLAS. Muscovite mica $\left(\mathrm{KAl}_{2}\left(\mathrm{AlSi}_{3} \mathrm{O}_{10}\right)(\mathrm{OH})_{2}\right)$ was purchased from Xinghua Guanjin Mica Products Factory and Nanoandmore. The surfaces of mica with various cations were characterized by atomic force microscopy (AFM) with a Multimode 8 (Bruker) and X-ray photoelectron spectroscopy (XPS) (ESCALab220i-XL).

\section{Methods}

Ice nucleation measurements. The heterogeneous ice nucleation (HIN) temperature $\left(T_{\mathrm{H}}\right)$ and the ice nucleation delay time $\left(t_{\mathrm{D}}\right)$ (Figure 2) were measured using an optical microscope (Nikon AZ100) coupled with a high-speed camera (Nikon Digital sight DSRi1). All samples were measured in a closed sample cell atop a cryostage (Linkam Scientific Instruments Ltd) ${ }^{2}$, as illustrated in Scheme S1. The sample cell was composed of two cover glasses upheld by a rubber O-ring. Inside the closed cell, one $0.1 \mu \mathrm{L}$ droplet of Milli-Q- $\mathrm{H}_{2} \mathrm{O}$ was deposited atop the freshly prepared mica surface. The sealed sample cell was then placed into the chamber of the cryostage and cooled at various cooling rates of 1.0, 2.0, 5.0 and $10{ }^{\circ} \mathrm{C} \mathrm{min}-1$ until the droplet was frozen. The freezing of the water droplet was observed through a microscope equipped with a highspeed camera. The $t_{\mathrm{D}}$ value of the ice nucleation was obtained at different supercooling temperatures with a cooling rate of $5.0{ }^{\circ} \mathrm{C} \mathrm{min}^{-1}$ in sealed cells with a relative humidity of $100 \%$ by fixing the surface temperature and recording the time interval between the time when the surface reached the target temperature and that when freezing occurred. The deposition of water droplets and the fabrication of the sealed sample cell were carried out in a vertical laminar flow clean bench to avoid possible contamination. The average values of $T_{\mathrm{H}}$ were obtained based on approximately 100 independent ice nucleation events atop different locations, and the error bars are the standard error on the mean. That is, for each type of ion-exchanged mica, the measurements were performed on $c a .100$ substrates, and one droplet of $0.1 \mu \mathrm{L}$ Milli-Q- $\mathrm{H}_{2} \mathrm{O}$ was deposited on top of each substrate. Note that the cleaved mica had to be carefully prepared by peeling the entire layer off to avoid possible defects. 
SFG measurements. Sum frequency (SF) generation is a second-order nonlinear process that provides interface-specific information due to its selection rules. ${ }^{3-5}$ An infrared (IR) beam (9 muJ pulse energy, central frequency of $3300 \mathrm{~cm}^{-1}$, FWHM of 350 $\mathrm{cm}^{-1}$, incident angle of $40^{\circ}$ to surface normal) in resonance with the stretch vibration of the water molecules is used and up-converted with a visible laser pulse (central wavelength $810 \mathrm{~nm}, 20 \mathrm{muJ}$ pulse energy, incident angle to surface normal of $70^{\circ}$ ) generating SFG pulses with a central frequency of $674 \mathrm{~nm}$. The vibrational spectrum of the SFG light is a marker of the strength of the local hydrogen bond network, and the intensity provides information about the order of alignment. ${ }^{6}$ In particular, the intensity of the SF light scales as $I \propto|N<\beta>|^{2}$ with the number of excited oscillators $N$ and an order parameter $\langle\beta>$ that is proportional to the average alignment of the water dipole moment $\left\langle\mu_{\mathrm{z}}>\right.$ in the case of an SSP polarized SF experiment.

The SF signal is very sensitive to the relocation of mica and varies from sample to sample (Figure S4). A flow cell was used to allow the exchange of the surface cation by flushing sequences of ionic solution and Milli-Q- $\mathrm{H}_{2} \mathrm{O}$. To exchange the surface cations of mica, the freshly cleaved mica was exposed to a flow of $0.5 \mathrm{M} \mathrm{HCl}, \mathrm{NaCl}$, $\mathrm{KCl}, \mathrm{RbCl}$ or $\mathrm{CsCl}$ solution for approximately 30 minutes. Subsequently, the ionic solution was removed from the flow cell. The mica surface was rinsed with a flow of Milli-Q- $\mathrm{H}_{2} \mathrm{O}$. Afterwards, the SF spectra of the interfacial water in contact with the cation-mica were measured at ambient temperature. This method allowed us to obtain all the relevant SF data without moving the sample cell and is the key to obtaining reproducible results.

Characterization of cation-embedded mica surfaces. The chemical composition of the cation-embedded mica surfaces was characterized by XPS (Figures S1-S2) to validate the cation exchange process and confirm the successful exchange of the cations to the mica surfaces. The morphology of the freshly prepared cation-embedded mica surfaces was characterized by AFM, as shown in Figure S3. The contact angle was measured with a DSA 100 (Krüss): $0.1 \mu \mathrm{L}$ water was deposited on the freshly prepared cation-exchanged mica surface with a microsyringe in an open system at ambient temperature. The image of the water droplet was taken by a CCD camera. Then, the 
contact angles were obtained by fitting the image of the $0.1 \mu \mathrm{L}$ water droplet using circle mode (Figure S4).

Molecular dynamics simulation. The HIN processes of water on the surfaces of mica were studied by MD simulation. In this work, water was described by a full-atomic potential TIP6 $\mathrm{P}^{7}$ with an ice melting point at $289 \mathrm{~K},{ }^{8}$ which has been used to successfully simulate both homogeneous and heterogeneous ice nucleation in previous studies. ${ }^{9-13}$ The CLAYFF ${ }^{14}$ potential has been widely used to study water on various clay mineral surfaces. In this work, mica was described by the CLAYFF potential with the parameters listed in Table S1. The cross interactions between water and mica were calculated by using the Lorentz-Berthelot rule. A slab model of mica (001) was created with lateral ( $\mathrm{x}$ and y) dimensions of $15.56 \AA \times 17.97 \AA$. A total of 384 water molecules were placed on top of the mica (001). Periodic boundary conditions were applied in the $x$-, $y$-, and $z$-directions, in which the $z$-direction is vertical to the surface. To avoid extra interactions, a large value (205.36 $\AA$ ), more than three times the height of the water and mica in the $z$-direction, was given for the box in the $z$-direction. The NVT ensemble with temperature controlled at $250 \mathrm{~K}$ by a Nosé-Hoover chain length of 10 and a time constant for temperature coupling of $0.5 \mathrm{ps}$ was employed in the MD simulations. The fast smooth particle-mesh Ewald method was used for the electrostatic interactions with a real-space cutoff of $6.5 \AA$. The van der Waals interactions were also truncated at 6.5 $\AA$. The leap-frog algorithm for integrating Newton's equations of motion with a time step of $1 \mathrm{fs}$ was selected in the MD simulations. Notably, the free hydrogen on the mica surface automatically moves toward and bonds with the oxygen in the DFT geometry optimization when $\mathrm{K}^{+}$is absent. In the $\mathrm{H}^{+}$exchanged mica, the $\mathrm{H}^{+}$is bonded with the nearest oxygen atom on the mica surface in the form of $\mathrm{OH}$ with a bond length of $1 \AA$. For ice nucleation simulations, a running time of $500 \mathrm{~ns}$ was usually set for each simulation. If we did not observe nucleation during $500 \mathrm{~ns}$, the trajectory was neglected, and then a new run was carried out with a different initial structure. All the classical MD simulations were performed using Gromacs 4.6.5 software.

Analysis of the dipole projection along the z-direction. As shown in Figure S12, the water molecules located in the region of $2.0 \mathrm{~nm}$ above the mica surface were selected for the dipole analysis. Dipole projection along the z-direction of each water molecule was computed, as depicted in Figure S19. To investigate the relations of the water 
orientations at the interface with the ionic size on the surface of the mica and the interactions between the water and mica, the trajectories with fixed $\sigma / \varepsilon$ and adjusted $\varepsilon / \sigma$ were obtained, e.g., $\sigma$ increasing from $1.6 \AA$ to $4.4 \AA$ with a step of $0.4 \AA$ when $\varepsilon$ is $0.5216 \mathrm{~kJ} / \mathrm{mol} ; \boldsymbol{\varepsilon}$ increasing from $0.02 \mathrm{~kJ} / \mathrm{mol}$ to $1.42 \mathrm{~kJ} / \mathrm{mol}$ with a step of $0.2 \mathrm{~kJ} / \mathrm{mol}$ when $\sigma$ is $3.25 \AA$. $\sigma$ and $\varepsilon$ are the parameters of the LJ potential, which are used to tune the ionic size and the interaction between the water and the ions on the mica surface. For each $\sigma$ and $\varepsilon$ parameter, five independent trajectories are carried out for $70 \mathrm{~ns}$ at $250 \mathrm{~K}$ by using TIP6P and at $240 \mathrm{~K}$ by using TIP4P/Ice. For each trajectory, the initial $10.0 \mathrm{~ns}$ was considered to be the equilibration time, and then structure snapshots were taken at a step of $1.0 \mathrm{~ns}$. The dipole $\mathrm{z}$ was computed by taking the average of the dipole of the 60 structure snapshots for each trajectory. The functions between dipole $\mathrm{z}_{\mathrm{z}}$ and $\boldsymbol{\sigma} / \boldsymbol{\varepsilon}$ were fitted after the dipole $e_{z}$ was calculated based on the trajectories of various $\sigma / \varepsilon$, as plotted in Figure S20a-d. Then, the dipole ${ }_{z}$ of the water on each mica surface with various ion replacements was computed based on the two functions and the experimental ionic size (as listed in Table S2) and the adsorption energy per water molecule for each case (Figure 4d).

Adsorption energies computed by density functional theory. The adsorption energies between the interfacial water and the mica surface were computed by the QUICKSTEP program in $C P 2 K$ software under the framework of DFT methods based on a model of three-layer ice $I h$ placed on top of the mica (001) surface, as depicted in Figure S21. A slab model with dimensions of $15.71 \AA \times 9.11 \AA \times 45.36 \AA$ in the $x$-, $y$ , and $z$-directions was constructed from the unit cell of muscovite mica for DFT computations, in which a $25 \AA$ vacuum was included in the $z$-direction. A structure of three-layer ice Ih with $48 \mathrm{H}_{2} \mathrm{O}$ was placed on the mica surface. The adsorption energy $E_{A}=\left(E_{\text {total }}-E_{\text {mica }}-E_{\text {ice }}\right) / N_{H 2 O}$, in which $E_{\text {total }}$ is the energy of the system with threelayer ice on the mica surface, $E_{\text {mica }}$ is the energy of the mica surface, $E_{i c e}$ is the energy of the three-layer ice, and $N_{H 2 O}$ is the number of water molecules in the three-layer ice. Both a GGA functional $\mathrm{PBE}^{15}$ without and with dispersion correction ${ }^{16}$ were employed in the geometry optimizations. Goedecker - Teter - Hutter (GTH) norm-conserving pseudopotentials were used. The electron density is expanded by a combination of the Gaussian DZVP basis set and auxiliary plane waves with an energy cutoff of $300 \mathrm{Ry}$. In addition, Mulliken analysis and electron density distribution calculations are 
performed for the H-mica and K-mica surfaces (see Figure S18), respectively, after full atom relaxation. Ab initio molecular dynamics (AIMD) simulations are carried out by using the PBE-D3 functional at $300 \mathrm{~K}$ on the H-mica and K-mica surfaces with $48 \mathrm{H}_{2} \mathrm{O}$ for $60 \mathrm{ps}$. 
(a)

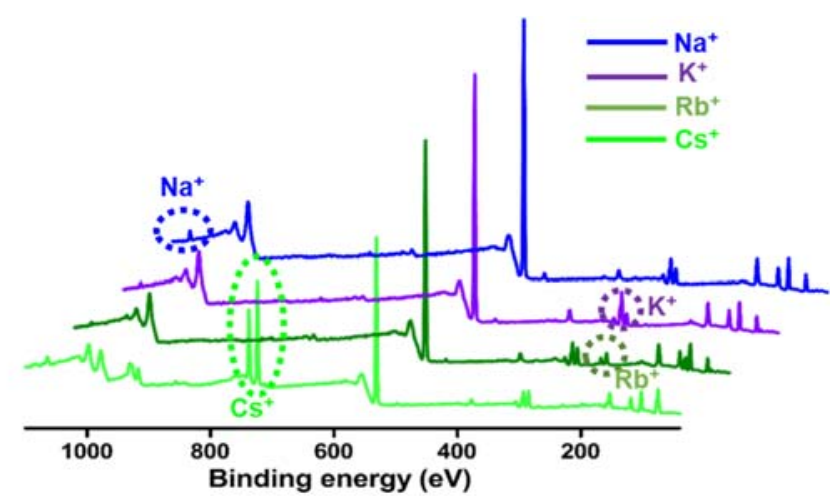

(b)

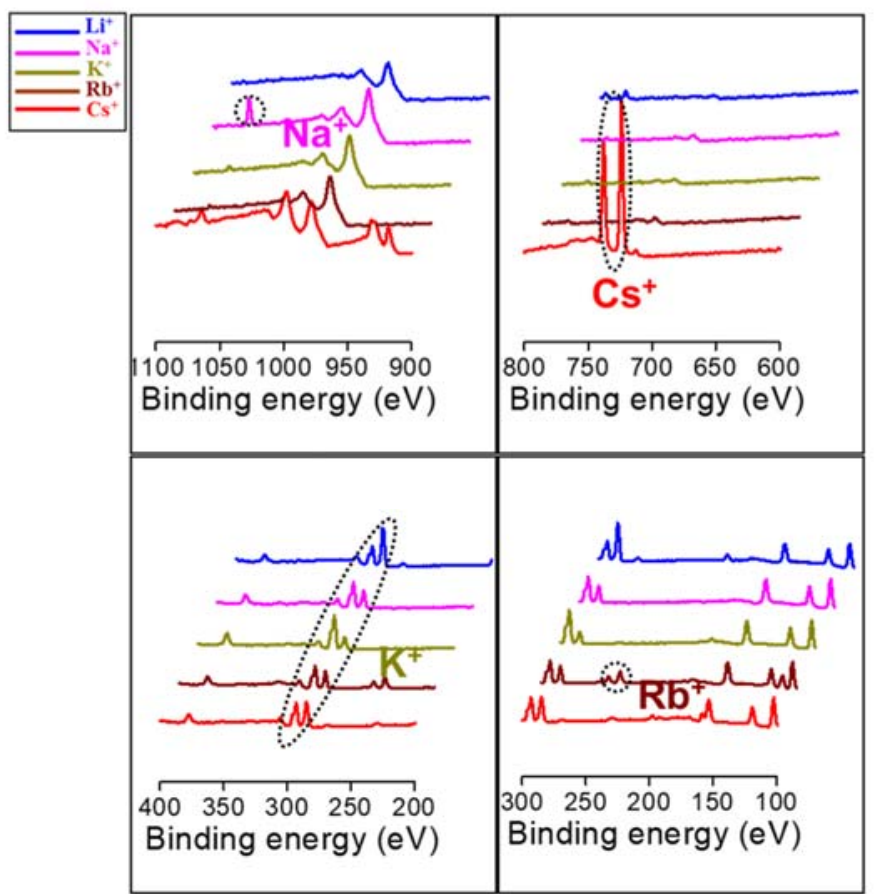

(c)

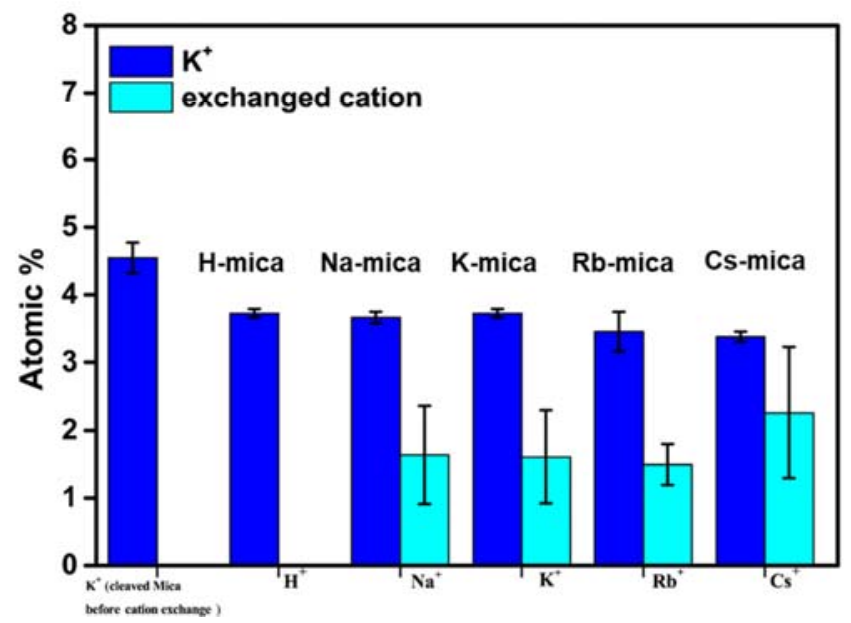

Figure S1. (a) The XPS spectra and (b) magnified XPS spectra verify the exchange of cations on the mica surfaces. (c) Quantitative analysis of the XPS results with each data point obtained based on average 3-5 measurements. The error bars represent \pm s.d. 
The ion exchange property of mica is limited to the surface ions, as the $\mathrm{K}^{+}$in the interlayers is not exchangeable under ambient conditions. ${ }^{17}$ Note that the $\mathrm{H}^{+}$in the $\mathrm{H}-$ mica does not produce signals in the XPS measurements, as reported by other groups. ${ }^{18,19}$ As shown in Figure S1c, the amount of $\mathrm{K}^{+}$on the freshly cleaved mica is ca. $0.8 \pm 0.16 \%$ higher than that on the $\mathrm{H}^{+}$-mica surfaces, which is considered the amount of $\mathrm{K}^{+}$left on the cleaved mica surface before cation exchange. Subtracting the amount of $\mathrm{K}^{+}$in the H-mica (ca. 3.7\%) from that in the K-mica (ca. 5.3\%) yields $c a$. $1.6 \%$ (i.e., the value of the cyan-colored column for K-mica), which is the amount of $\mathrm{K}^{+}$on the original outermost mica surface, i.e., nearly two times the amount of $\mathrm{K}^{+}$left on the cleaved mica $\left(c a .5 .3 \%-4.5 \%=0.8 \%\right.$, the amount of $\mathrm{K}^{+}$in the cleaved mica is $4.5 \%$ ), which corresponds with previous reports. ${ }^{18,20}$ The cyan columns indicate the amount of exchanged cations. The amounts of $\mathrm{Na}^{+}, \mathrm{K}^{+}, \mathrm{Rb}^{+}$and $\mathrm{Cs}^{+}$on the cationexchanged mica surfaces are $(1.64 \pm 0.73) \%,(1.61 \pm 0.69) \%,(1.50 \pm 0.30) \%$ and $(2.26 \pm 0.97) \%$, respectively. These data unambiguously confirm the successful exchange of cations on the mica surfaces, although the $\mathrm{K}^{+}$peak remains for all samples. 


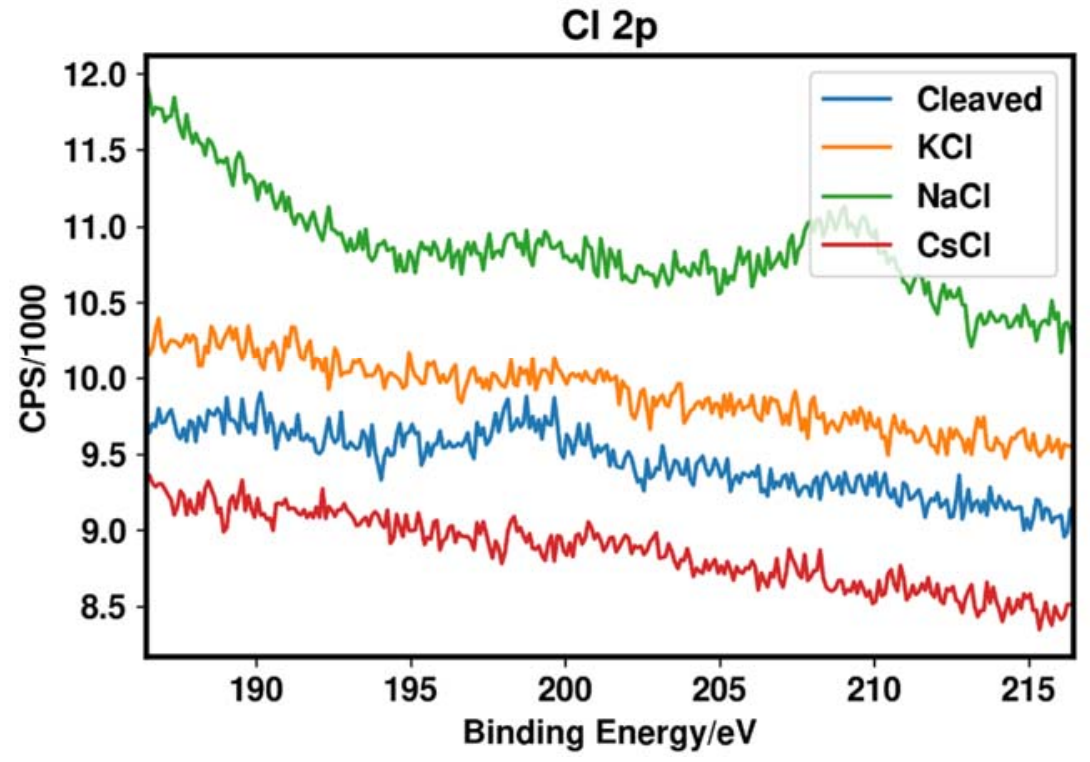

Figure S2. XPS of the $\mathrm{Cl} 2 \mathrm{p}$ orbital region on the freshly cleaved mica sample and mica samples exposed to $\mathrm{KCl}, \mathrm{NaCl}$ and $\mathrm{CsCl}$. There are no significant $\mathrm{Cl}$ signals, which should be present at $\sim 198 \mathrm{eV}$, for any of the samples. 


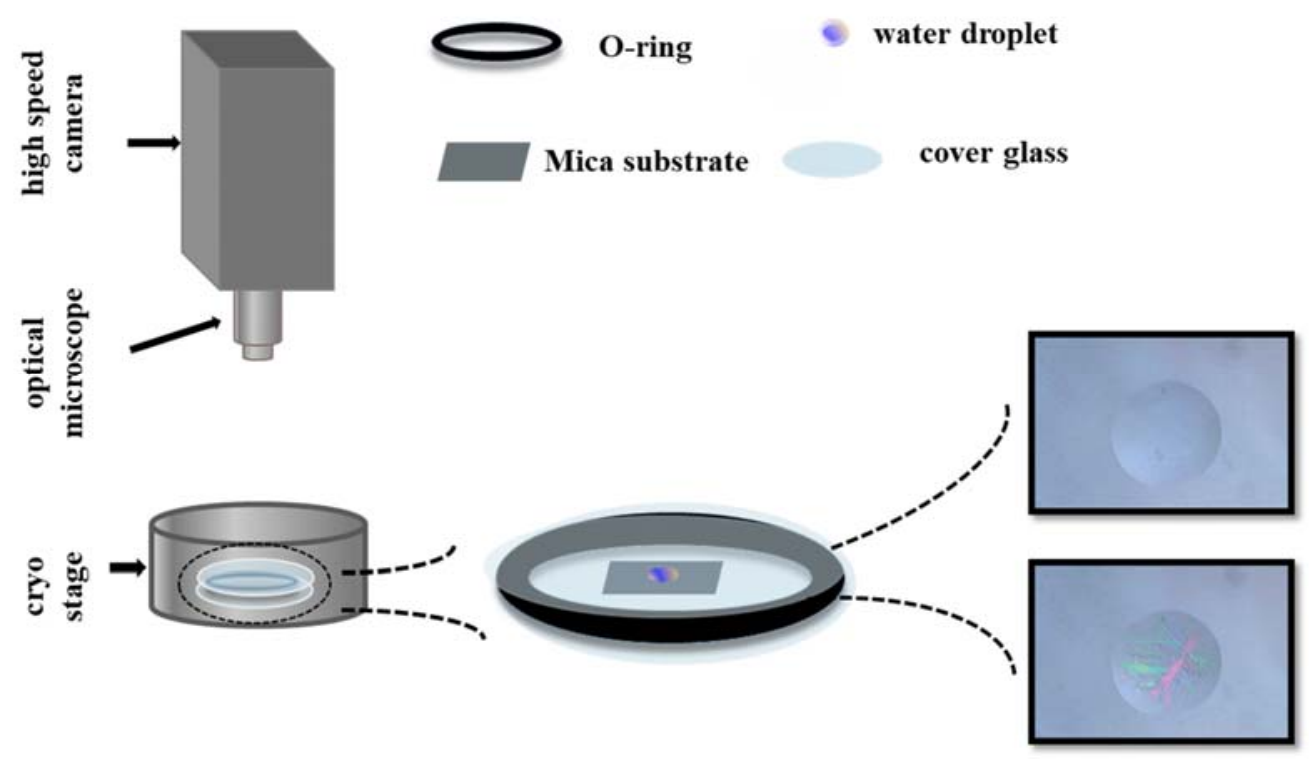

Scheme S1. Schematic representation of the experimental apparatus used to measure the heterogeneous ice nucleation temperature $\left(T_{\mathrm{H}}\right)$ on the cation-embedded mica surfaces. 
(a)
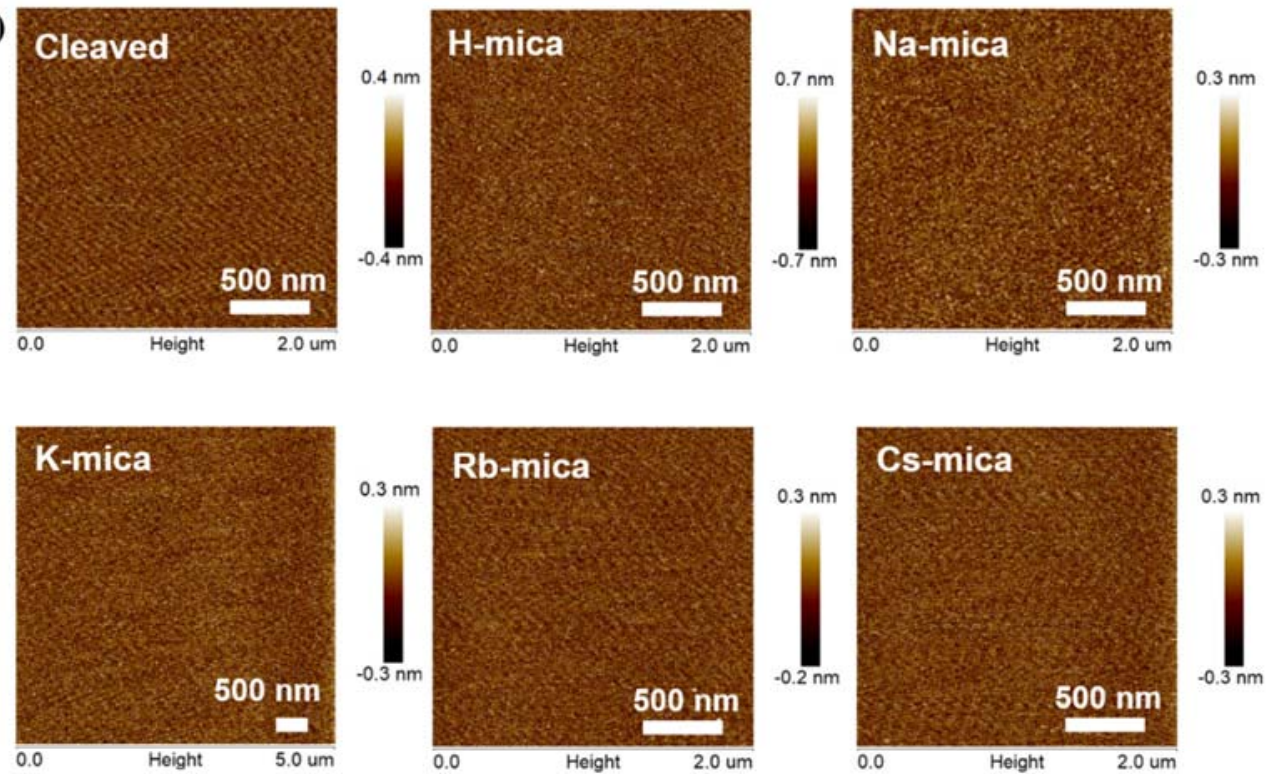

(b)

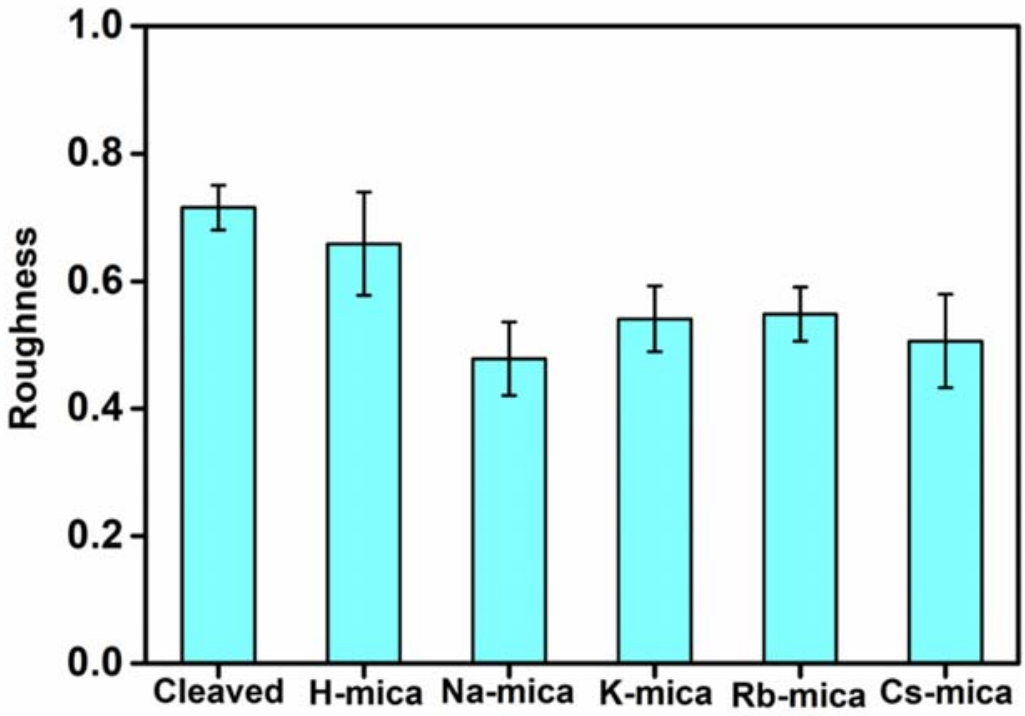

Figure S3. AFM characterization of the surface morphology and roughness of the cation-embedded mica surfaces. The surface (a) morphology and (b) roughness of the various cation-embedded mica surfaces determined by AFM. Error bars represent \pm s.d.

As shown in Figure S3a, no significant differences could be identified in the morphologies of the samples. Moreover, the roughness values of various cationexchanged mica surfaces were measured based on AFM, as shown in Figure S3b, which are less than $0.8 \mathrm{~nm}$. 
(a)

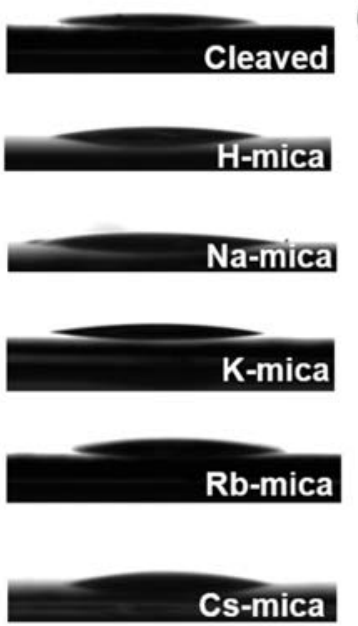

(b)

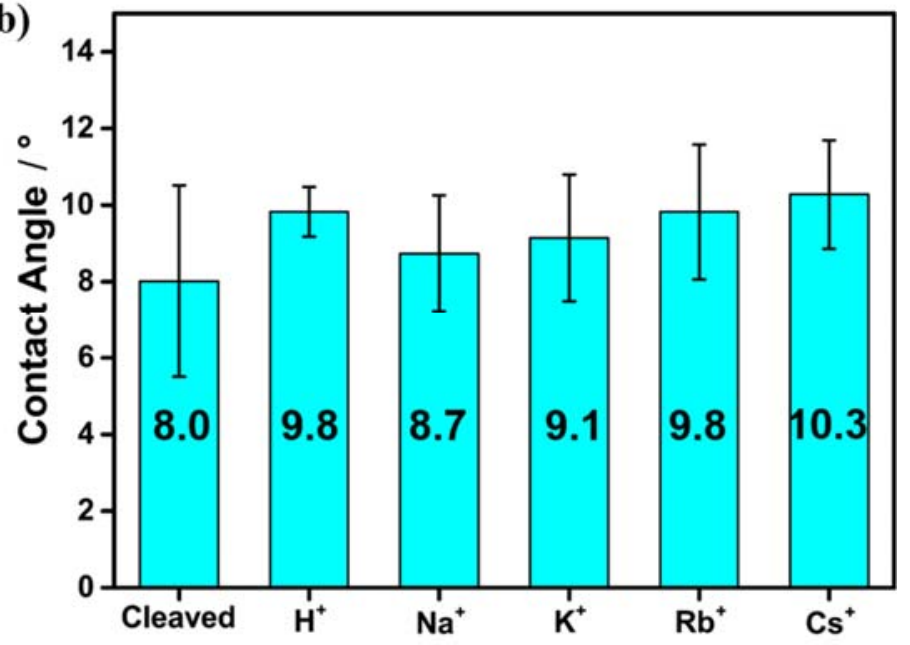

Figure S4. Characterization of the surface wettability of the cation-embedded mica surfaces. (a) Contact angle images of various cation-embedded mica surfaces. (b) The values of the contact angles on various cation-embedded mica surfaces are approximately $8.0 \sim 10^{\circ}$. The error bars represent \pm s.d.

The contact angles of $0.1 \mu \mathrm{L}$ water on the cation-exchanged mica surfaces are shown in Figure S4. The contact angles on various cation-exchanged mica surfaces are approximately $\left(8.0 \sim 10^{\circ}\right) \pm\left(0.65 \sim 2.5^{\circ}\right)$, which suggests that the contact patches of $0.1 \mu \mathrm{L}$ water on various cation-embedded mica surfaces are nearly the same within error. 


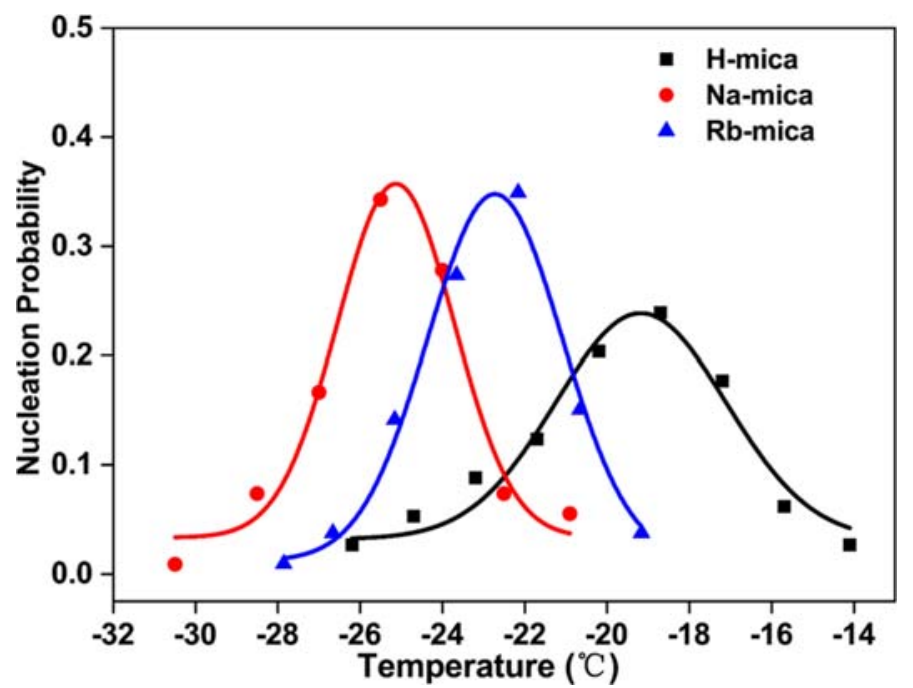

Figure S5. Ice nucleation probability distributions with Gaussian fitting calculation. 

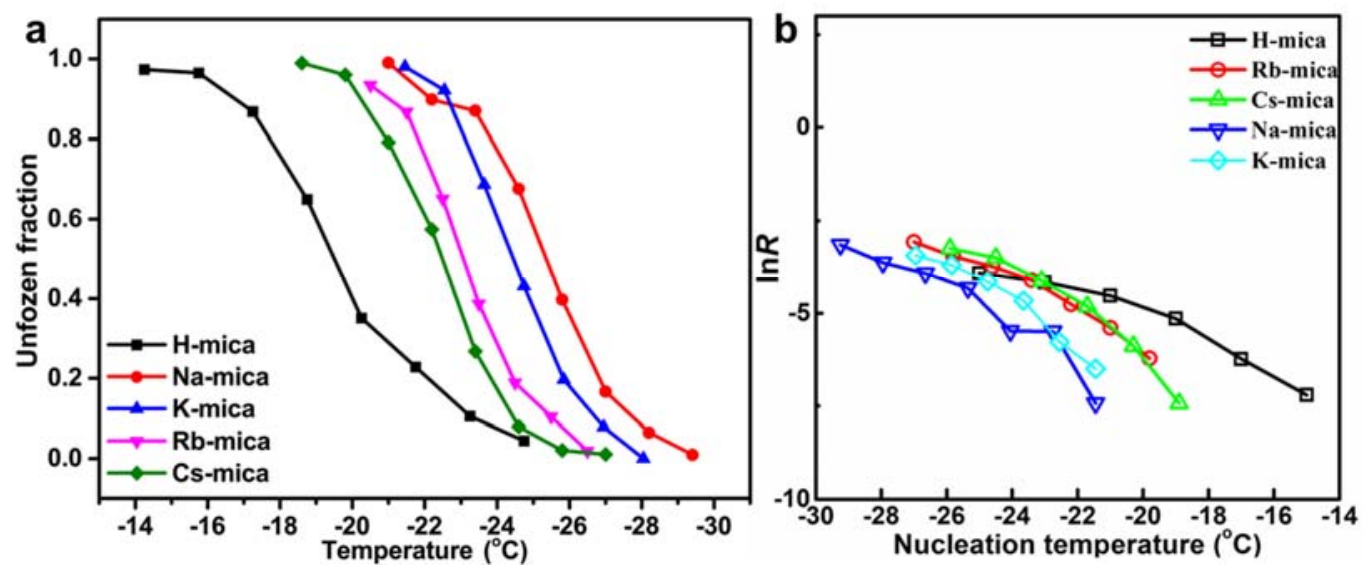

Figure S6. (a) Fraction of water droplets unfrozen as a function of temperature for various cation-embedded mica surfaces. (b) Freezing rates calculated for various cation-embedded mica surfaces.

The fraction of water droplets unfrozen as a function of temperature for various mica surfaces is shown in Figure S6a. The unfrozen fraction is calculated from $n_{\mathrm{i}}$ droplets frozen from a population of $n_{\mathrm{L}}$, which is written $\mathrm{as}^{21,22}$ :

$P=1-\frac{n_{i}}{n_{L}}$

The freezing rate $(R)$ per unit time (Figure S6b) was calculated based on equation (2): $R=\frac{-\ln \left(1-\frac{n_{i}}{n_{L}}\right)}{\Delta t}$

where $R$ is calculated from the unfrozen fraction $P$ during the time period $\Delta t .^{21,22}$ In general, lower freezing rates were accessed for Na-mica and K-mica, and H-mica possessed the highest freezing rate. 


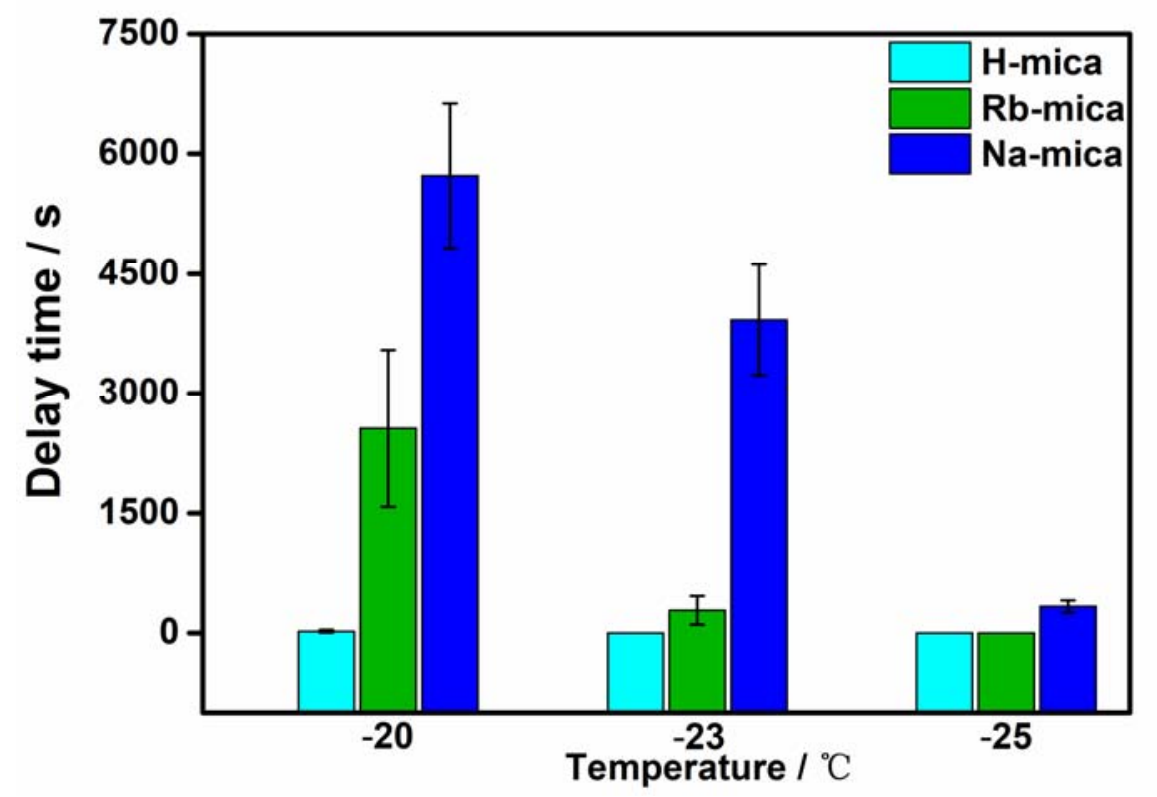

Figure S7. The ice nucleation delay time $\left(t_{\mathrm{D}}\right)$ of water droplets on H-mica, Na-mica and Rb-mica (001) at various supercooling temperatures. 


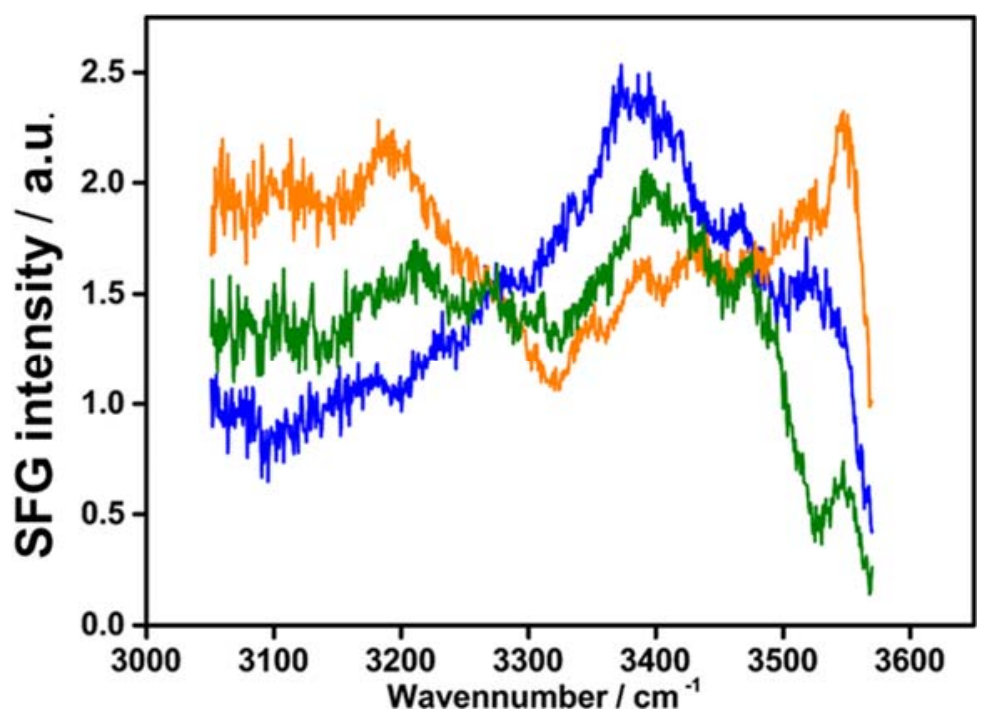

Figure S8. Static SF signals of interfacial water on different mica samples.

The SF spectra of the mica- $\mathrm{H}_{2} \mathrm{O}$ interface of 3 different mica samples are shown in Figure S8. The signal between $3000 \mathrm{~cm}^{-1}$ and $3550 \mathrm{~cm}^{-1}$ originates from water $\mathrm{H}$-bond stretching vibrations at the mica-water interface. The differences in the spectral shape are due to sample-to-sample variations. First, note that the SF spectra were obtained in a buried geometry, and thus, the thickness of the specific mica sample 0:15 mm - 0:21 $\mathrm{mm}$ plays a role, as the laser beams need to penetrate this thickness. Second, mica is a natural mineral and as such contains impurities. Third, the hydroxyl group shows a rotational anisotropy ${ }^{23}$ that we also observed that affects the H-bonded region of the water signal. However, this effect is out of the scope of this work and irrelevant, as we are using a flow cell to exchange the ions and deduce the results from the changes in the relative intensity before and after ion exchange on the same sample and in the exact same geometry. 


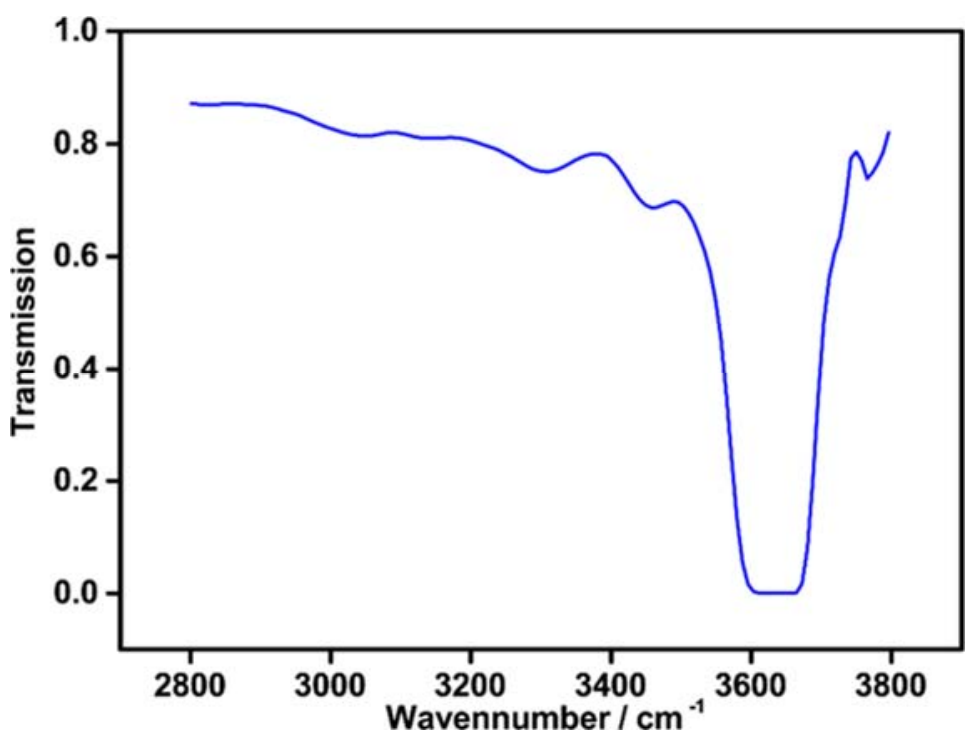

Figure S9. IR Transmission of a mica sample.

Figure S9 shows a strong absorption at approximately $3650 \mathrm{~cm}^{-1}$. This absorption is known to originate from the hydroxyl groups within the lattice structure and is why the SF spectra drop rapidly above $3550 \mathrm{~cm}^{-1}$. Furthermore, most of the spectrum is flat, except for some fluctuations at approximately $3400 \mathrm{~cm}^{-1}$. 


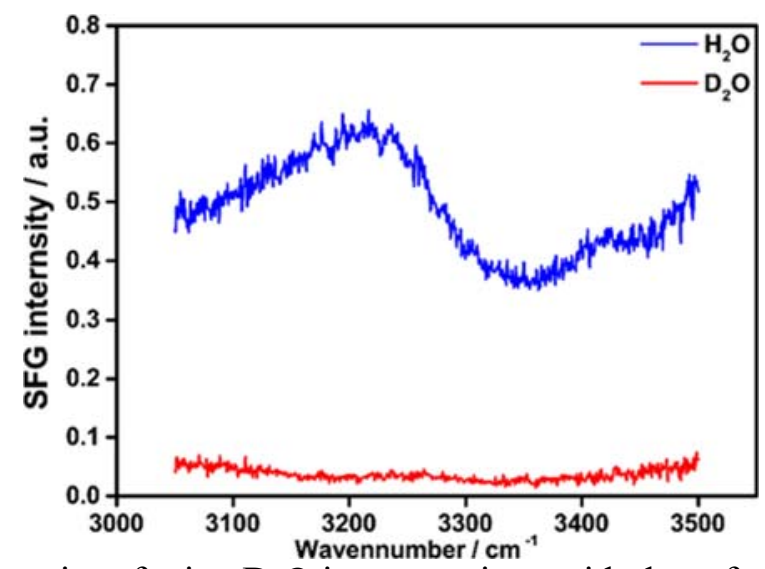

Figure S10. SF intensity of mica- $\mathrm{D}_{2} \mathrm{O}$ in comparison with that of mica- $\mathrm{H}_{2} \mathrm{O}$ in the $\mathrm{OH}$ stretch region. The signal of the bonded region from $3100 \mathrm{~cm}^{-1}$ to $3500 \mathrm{~cm}^{-1}$ vanishes upon isotopic exchange.

Figure $\mathrm{S10}$ shows the SF from the mica- $\mathrm{H}_{2} \mathrm{O}$ (blue) and mica- $\mathrm{D}_{2} \mathrm{O}$ (orange). The almost zero signal for the nonresonant $\mathrm{D}_{2} \mathrm{O}$ case indicates that the signal in the case of mica- $\mathrm{H}_{2} \mathrm{O}$ originates from noncentrosymmetric interfacial $\mathrm{H}_{2} \mathrm{O}$. 

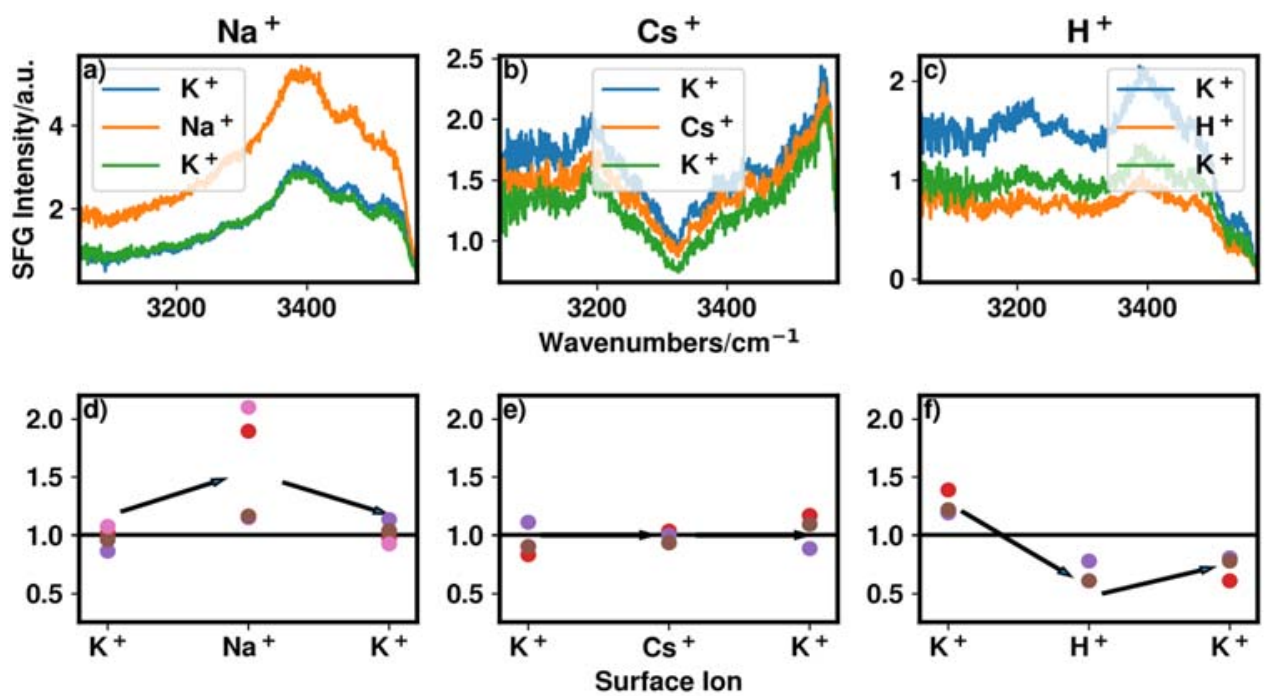

Figure S11 a-c SF spectra of the mica-water interface. Each panel shows a spectrum of the mica surface with initial $\mathrm{K}^{+}$in blue, the spectra of the mica surface with $\mathrm{Na}^{+}, \mathrm{Cs}^{+}$ and $\mathrm{H}^{+}$in orange and reversible exchange of $\mathrm{Na}^{+}, \mathrm{Cs}^{+}$and $\mathrm{H}^{+}$with $\mathrm{K}^{+}$in green. In comparison to the SF signal of K-mica, from left to right, Na-mica enhances the SF signal; Cs-mica barely changes the SF signal; and the H-mica surface significantly decreases the SF signal. d-f Trends of repeated SF intensity measurements as relative variation in Na-mica, Cs-mica, and H-mica.

Figure S11a shows the SFG spectra of the mica-water interface of Na-mica, Cs-mica and H-mica. The subsequent recording of a second K-mica spectrum after the measurement confirms the differences in the SFG signals caused by ion-specific effects. Figure S11a shows an enhancement of more than a factor of 2 in the amplitude of the SFG intensity of the mica interfacial water signal on the Na-mica. Figure S11b shows that the mica interfacial water on the Cs- and K-mica exhibits the same SFG signal intensity within the experimental uncertainty. This suggests that these ions have similar effects on mica interfacial water. Figure S11c illustrates the influence of H-mica on the interfacial water in contact with the mica relative to that of K-mica. A significant decrease is observed in the SFG intensity upon exchanging $\mathrm{K}^{+}$with $\mathrm{H}^{+}$at the mica surface.

Figure S11d-e shows the integrated SF intensities relative to the average SF intensity of the K-mica. Independent measurement sets are in the same color, and the surface ion content is denoted on the X-axis in temporal order. Arrows guide the eye toward significant trends. Especially in the case of Na-mica, two distinct cases are present, one in which the signal intensity increases almost by a factor of two and one where it increases by only approximately $20 \%$.

To cope with the sample-to-sample variations, we do not compare the absolute signal intensities but rather the changes in intensity due to the exchange of surface ions relative to the average K-mica. For example, we calculate

Relative SFG Intensity $==\frac{\sum, I_{S F, X}}{\left\langle\sum I_{S F, K+>}\right.}$

where $\sum I_{S F, X}$ is the summed intensity from $3000 \mathrm{~cm}^{-1}$ to 3550 of the ion $x \mathcal{E}\left[\mathrm{K}^{+}, \mathrm{Na}^{+}\right.$, 
$\left.\mathrm{Cs}^{+}, \mathrm{H}^{+}\right]$and $\iota$ denotes the average of the $\mathrm{K}^{+}$spectra before and after ion exchange. 
(a)

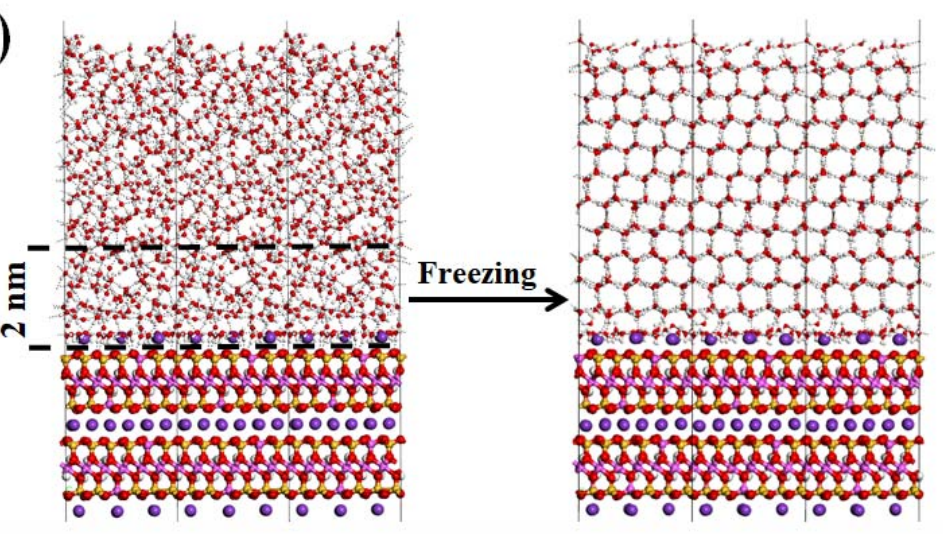

(b)

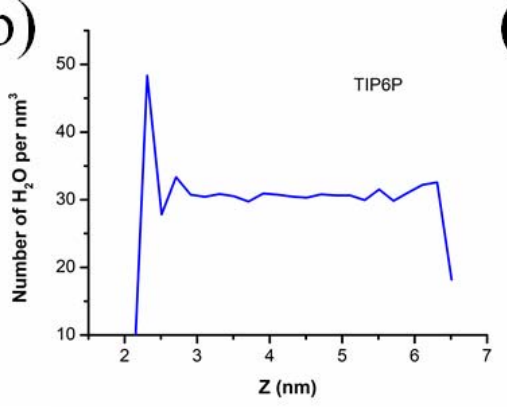

(c) ${ }^{5}$

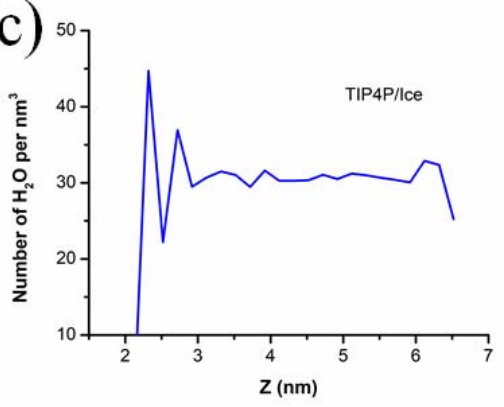

(d)

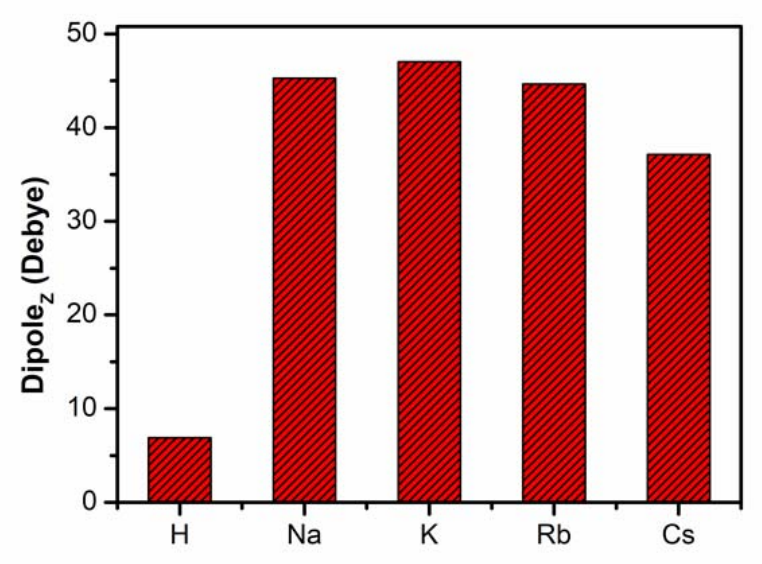

Figure S12. (a) Structure model of water on the mica (001) surface. Water molecules in the $2 \mathrm{~nm}$ region atop the mica surface are sampled for dipole analysis. (b) Density profile of the number of water molecules per $\mathrm{nm}^{3}$ along the z-direction (normal to the mica surface) with the TIP6P water potential. (c) Density profile of the number of water molecules per $\mathrm{nm}^{3}$ along the z-direction (normal to the mica surface) with the TIP4P/Ice water potential. (d) The dipole projections along the z-direction for each case with the TIP4P/Ice water potential. Note: the top layer of the mica surface is at the position 2 $\mathrm{nm}$ in the z-direction (average z-position of the oxygen atoms of the top layer of mica). 
(a)

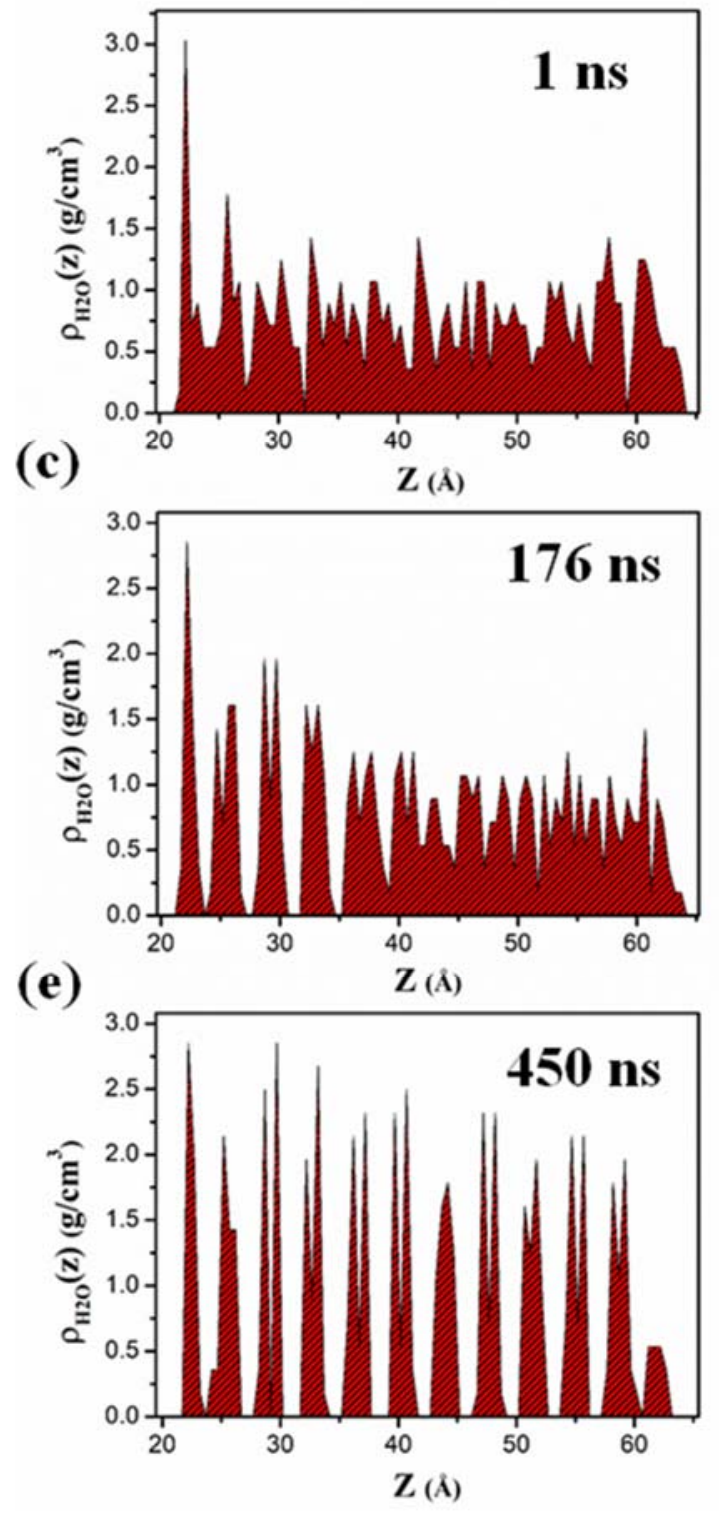

(b)

(d)
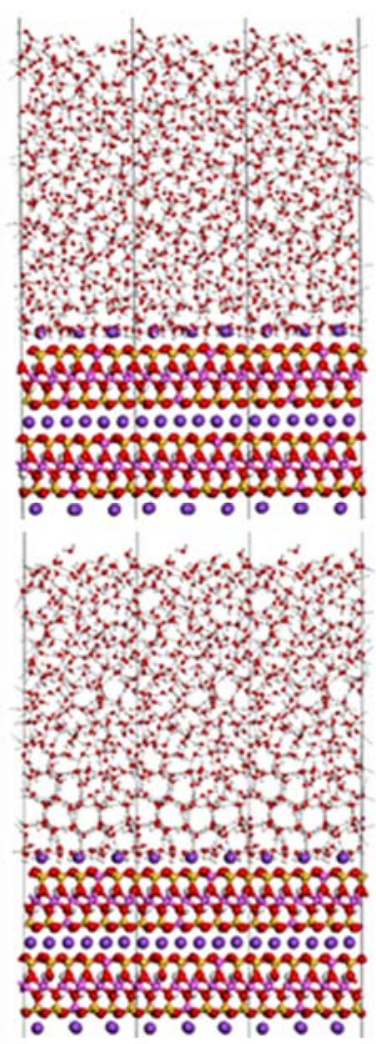

(f)

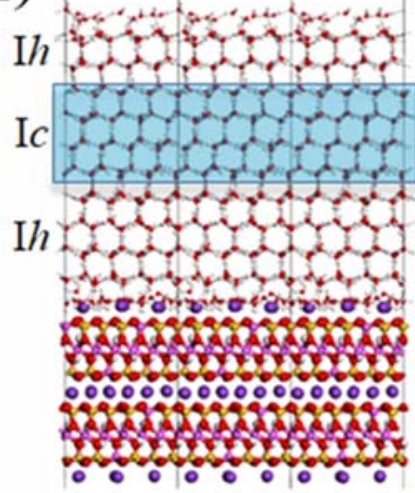

Figure S13. HIN on the surface of K-mica (001). (a, c, e) Density distribution along the z-direction and (b, d, and f) structure snapshots corresponding to 1, 176, and 450 ns. Note: the yellow balls represent $\mathrm{Si}$ atoms, red balls represent oxygen atoms, white balls represent hydrogen atoms, pink balls represent $\mathrm{Al}$ atoms, and light purple balls represent $\mathrm{K}$. I $c$ and $\mathrm{I} h$ indicate the type of ice structure. 
(a)

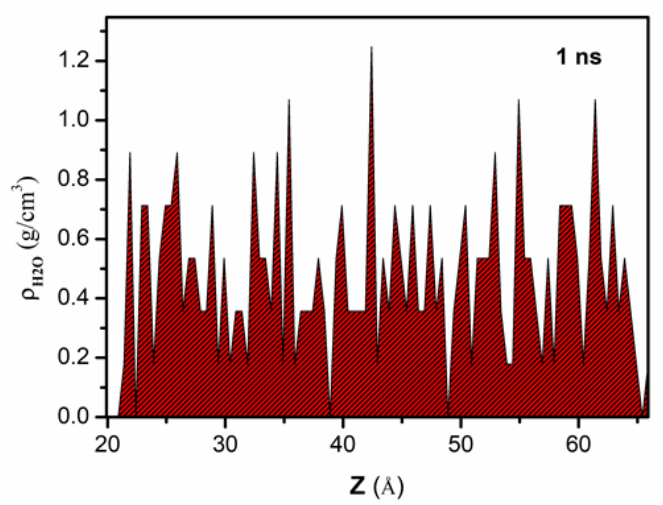

(c)

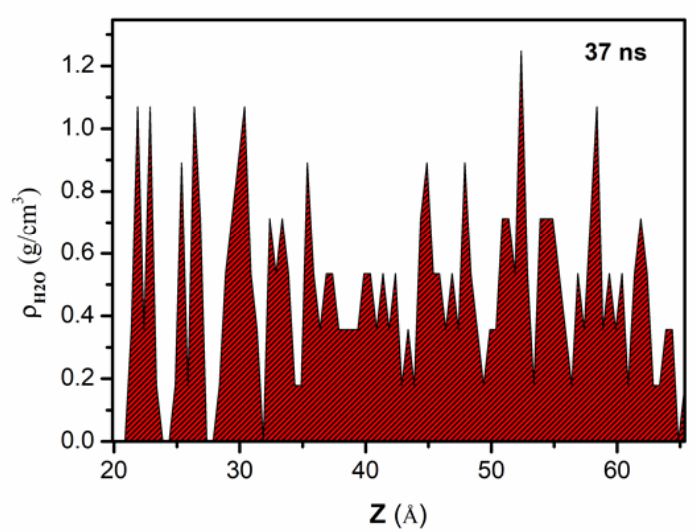

(e)

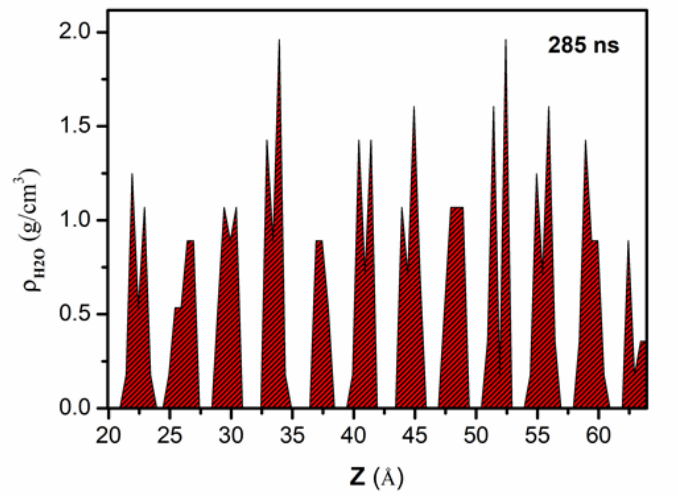

(b)

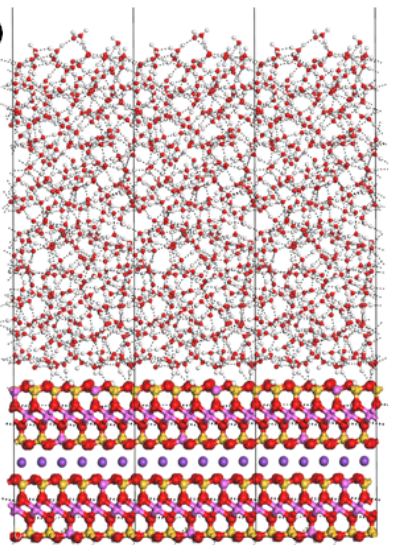

(d)

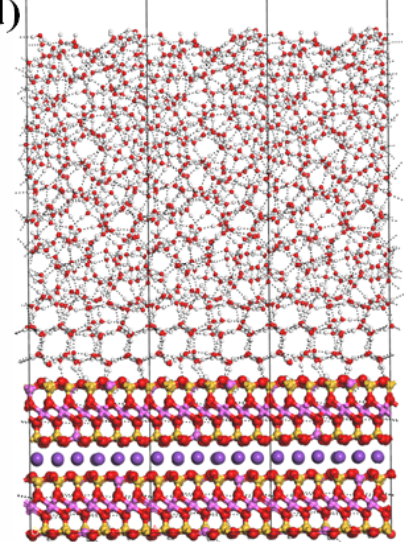

(f)

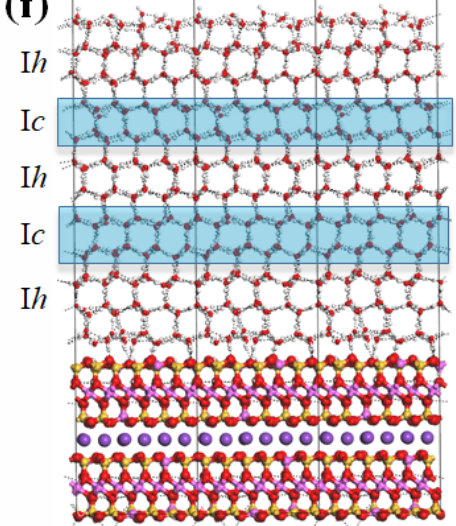

Figure S14. HIN on the surface of the H-mica (001). (a, c, e) Density distribution along the z-direction and (b, d, and f) structure snapshots corresponding to 1, 37, and $285 \mathrm{~ns}$. Note: the yellow balls represent $\mathrm{Si}$ atoms, red balls represent oxygen atoms, white balls represent hydrogen atoms, pink balls represent $\mathrm{Al}$ atoms, and light purple balls represent K. I $c$ and $\mathbf{I} h$ indicate the type of ice structure. 
Table S1. Parameters for mica used in the molecular dynamics simulation.

\begin{tabular}{|l|l|l|l|l|l|}
\hline Symbol & Charge $(\mathrm{e})$ & $\boldsymbol{\sigma}(\mathrm{nm})$ & $\boldsymbol{\varepsilon}(\mathrm{kJ} / \mathrm{mol})$ & Symbol & Charge (e) \\
\hline Ho & 0.42 & 0.0 & 0.0 & Ho & 0.43 \\
\hline Alt & 1.83 & 0.37064 & $7.7 \times 10^{-6}$ & Alt & 1.83 \\
\hline Alo & 1.75 & 0.47943 & $5.5638 \times 10^{-6}$ & Alo & 1.75 \\
\hline Si & 2.1 & 0.37064 & $7.7 \times 10^{-6}$ & Si & 2.203333 \\
\hline Obos & -1.01 & 0.35532 & 0.6502 & Obos & -1.03 \\
\hline Obss & -1.135 & 0.35532 & 0.6502 & Obss & -1.15 \\
\hline Obts & -1.175 & 0.35532 & 0.6502 & Obts & -1.17 \\
\hline K & 1.14 & 0.325 & 0.5216 & K & 1.22 \\
\hline Na/K/Rb/Cs & 1.14 & $0.2876 / 0.325 / 0.3348 / 0.3526$ & 0.5216 & H & 0.54 \\
\hline
\end{tabular}

Note: The charges were obtained from the Milliken atomic populations based on DFT computations by using the PBE functional in the CASTEP program. The parameters of the Lennard-Jones potential were obtained from the CLAYFF of Cygan et al. (J. Phys. Chem. B 2004, 108, 1255-1266), and the LJ parameters of the alkali cations were taken from the work of Koneshan et al. (J. Phys. Chem. B 1998, 102, 4193-4204). In addition, Ho represents hydroxyl hydrogen atoms, Alt/Alo represents tetrahedral/octahedral aluminum, Si represents silicon atoms, and Obos/Obss/Obts represents bridging oxygen with octahedral/tetrahedral/double substitution. $\mathbf{K}$ represents the $\mathrm{K}^{+}$in the bulk. $\mathbf{N a} / \mathbf{K} / \mathbf{R b} / \mathbf{C s}$ represents $\mathrm{Na}^{+} / \mathrm{K}^{+} / \mathrm{Rb}^{+} / \mathrm{Cs}^{+}$on the surface of mica. $\mathbf{H}$ represents the surface $\mathrm{K}^{+}$replaced by $\mathrm{H}^{+}$, which is bonded with a nearest oxygen atom on the mica surface in the form of $\mathrm{OH}$ with a bond length of $1 \AA$.

Table S2. Ionic radius of each ion taken from John Emsley, The Elements, $3^{\text {rd }}$ edition. Oxford: Clarendon Press, 1998.

\begin{tabular}{|l|l|l|l|l|l|}
\hline Ions & $\mathrm{Li}^{+}$ & $\mathrm{Na}^{+}$ & $\mathrm{K}^{+}$ & $\mathrm{Rb}^{+}$ & $\mathrm{Cs}^{+}$ \\
\hline Ionic radius $(\AA)$ & 0.78 & 0.98 & 1.33 & 1.49 & 1.65 \\
\hline
\end{tabular}


(a)

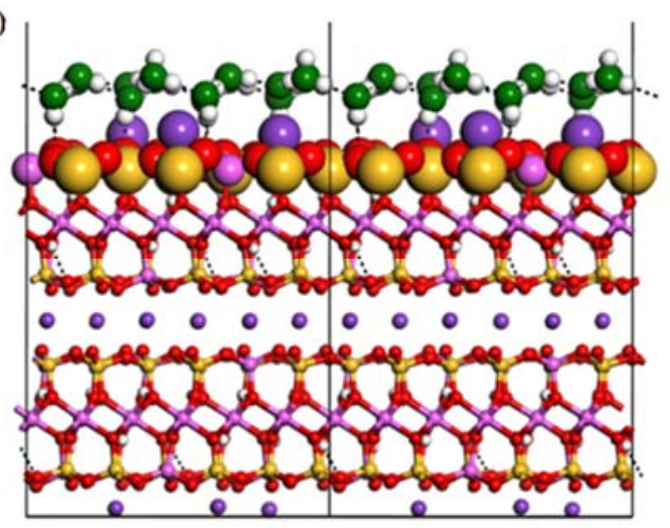

(b)

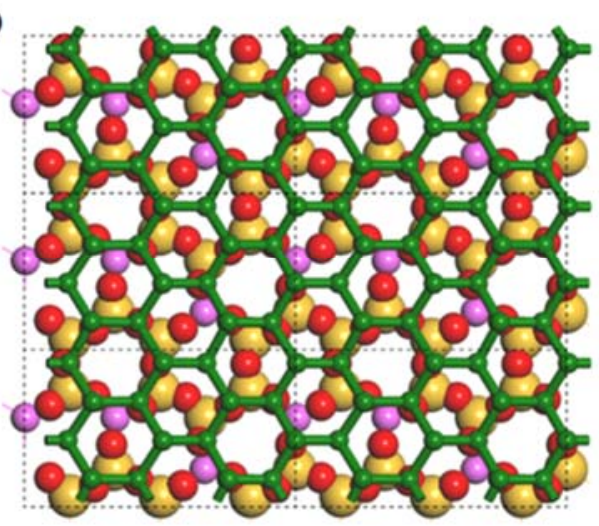

Figure S15. A layered ice structure where the basal face of ice $\mathrm{I} h$ is placed on top of mica (001) without any optimization, (a) side view, (b) top view. Color scheme: the yellow balls represent $\mathrm{Si}$ atoms, red balls represent the oxygen atoms of mica, white balls represent hydrogen atoms, pink balls represent $\mathrm{Al}$ atoms, light purple balls represent $\mathrm{K}^{+}$, and green balls on the top represent the oxygen atoms of water. The $\mathrm{K}^{+}$ and the hydrogen atoms of water are not pictured in (b). 


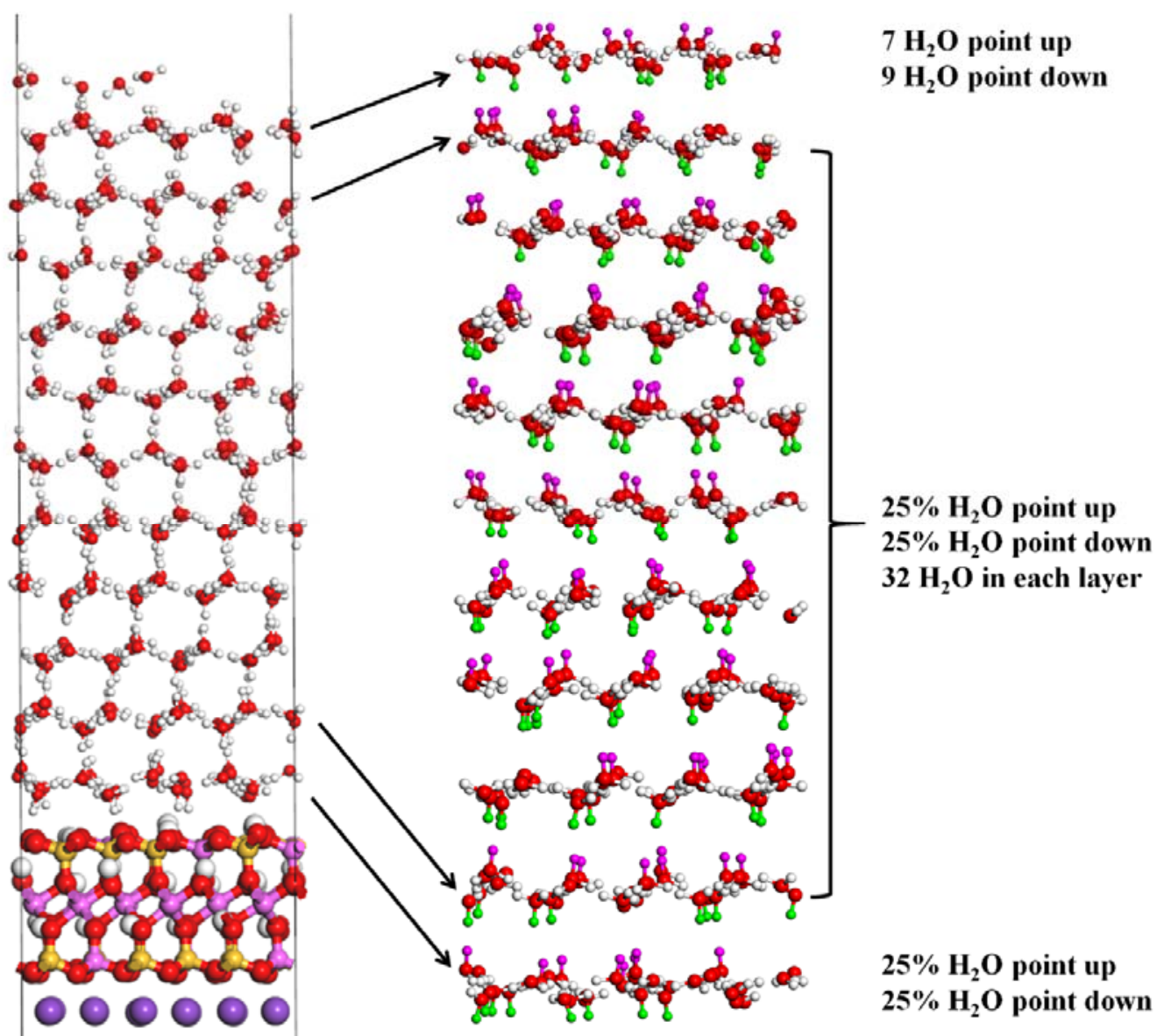

Figure S16. Structure snapshot of water on the H-mica surface after freezing was complete. Color scheme: the yellow balls represent Si atoms, red balls represent oxygen atoms, white balls represent hydrogen atoms, pink balls on the left represent $\mathrm{Al}$ atoms, and light purple balls represent $\mathrm{K}$. To show the specific water orientation, the hydrogen atoms of the water molecules pointing up are marked in pink and the hydrogen atoms of the water molecules pointing down are marked in green. 


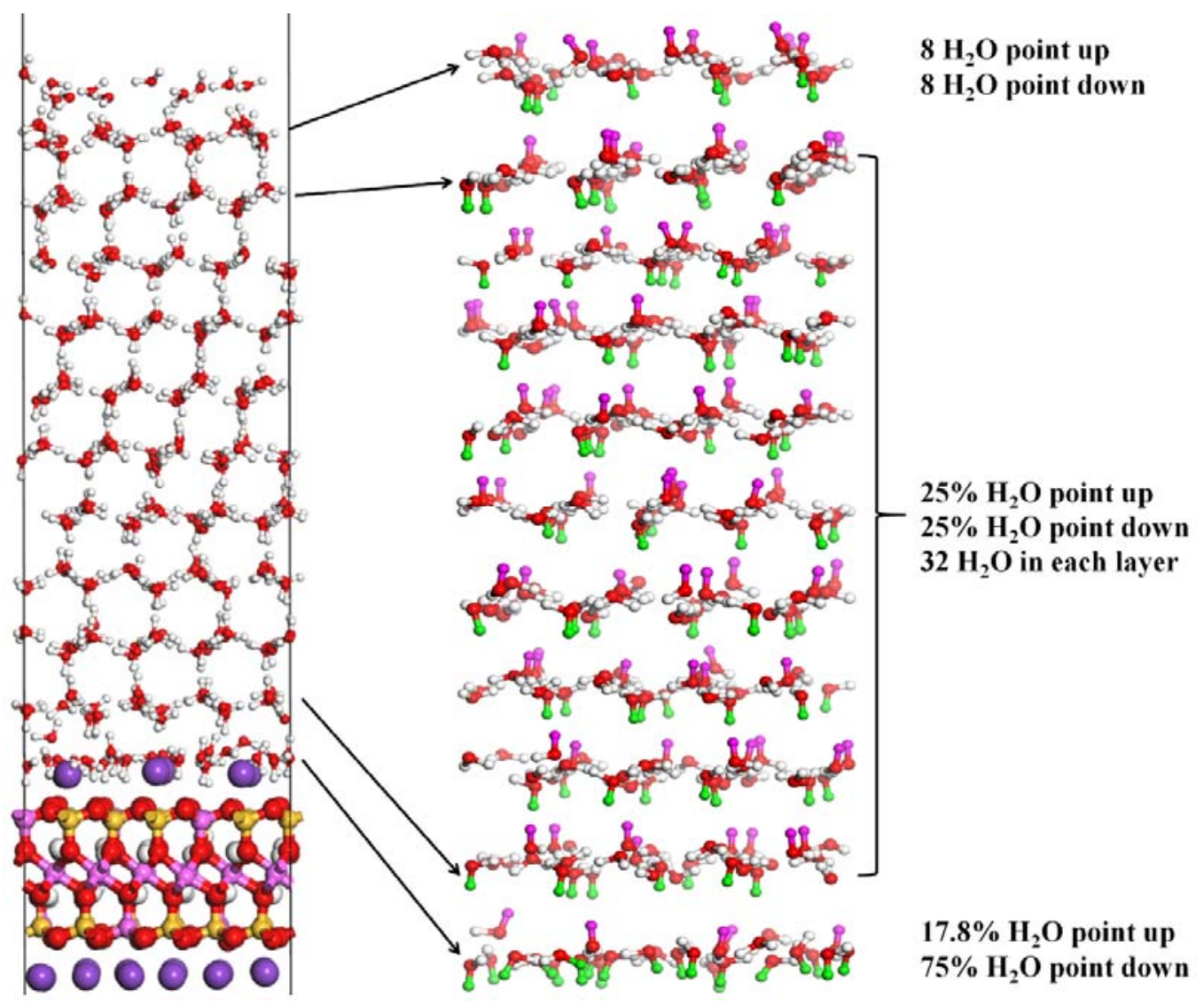

Figure S17. Structure snapshot of water on the K-mica surface after freezing was complete. Color scheme: the yellow balls represent $\mathrm{Si}$ atoms, red balls represent oxygen atoms, white balls represent hydrogen atoms, pink balls on the left represent $\mathrm{Al}$ atoms, and light purple balls represent $\mathrm{K}$. To show the specific water orientation, the hydrogen atoms of the water molecules pointing up are marked in pink and the hydrogen atoms of the water molecules pointing down are marked in green. 
(a)

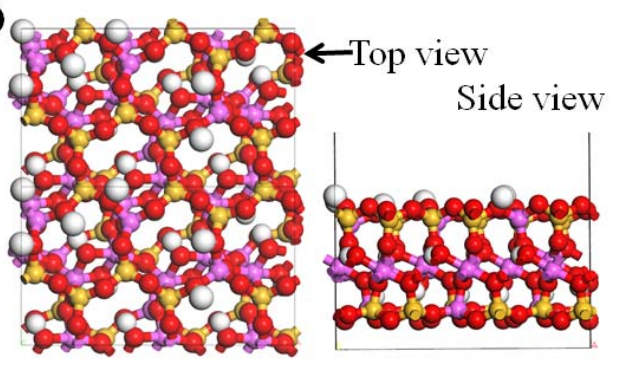

(b)

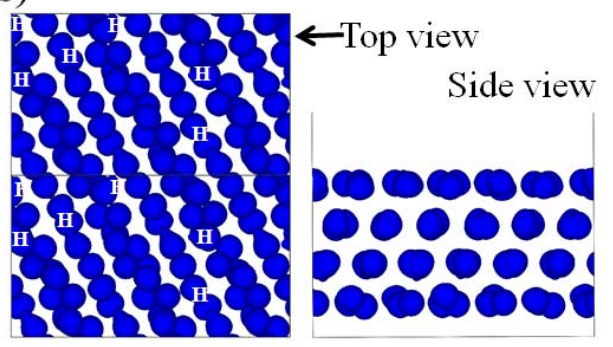

\section{H-terminated mica}
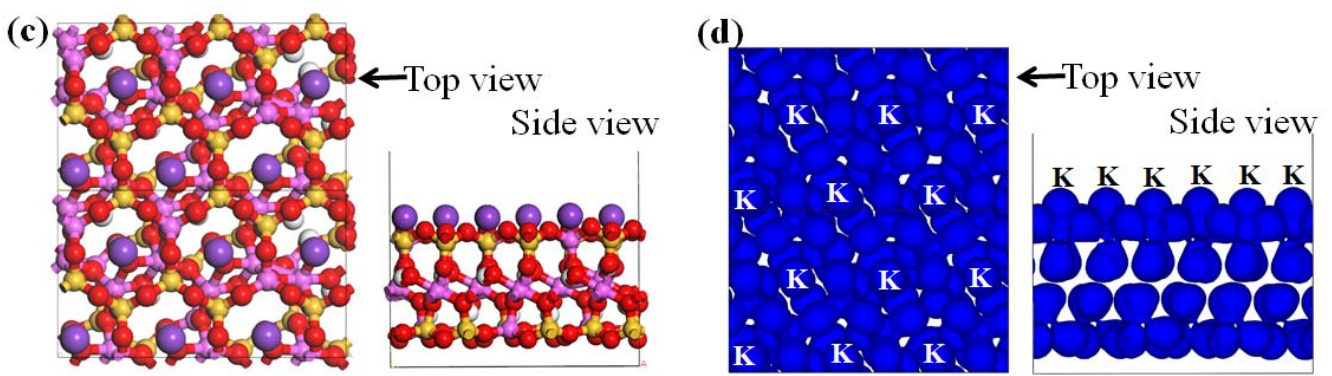

K-terminated mica

Figure S18. Structure model of the H/K-mica surface and the corresponding electron density distribution. (a) Top view and side view of the H-mica model, (b) top view and side view of the electron density distribution of the H-mica surface, (c) top view and side view of the K-mica model, and (d) top view and side view of the electron density distribution of the K-mica surface. Color scheme: the yellow balls represent Si atoms, red balls represent oxygen atoms, white balls represent hydrogen atoms, pink balls on the left represent $\mathrm{Al}$ atoms, and light purple balls represent $\mathrm{K}$. The blue color in (b) and (d) indicates the isosurface of the electron density distribution at the same level. 


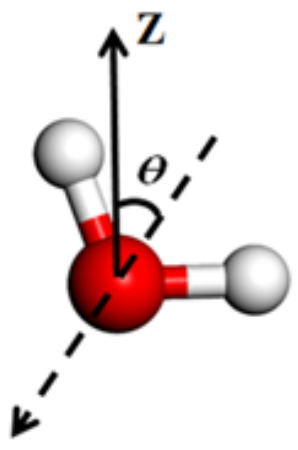

\section{Dipole $_{\mathrm{z}}=$ Dipole $_{\mathrm{H} 2 \mathrm{O}} \times \cos (\pi-\theta)$}

Figure S19. The dipole projection along the z-direction is calculated according to the specific water orientation. 
(a)

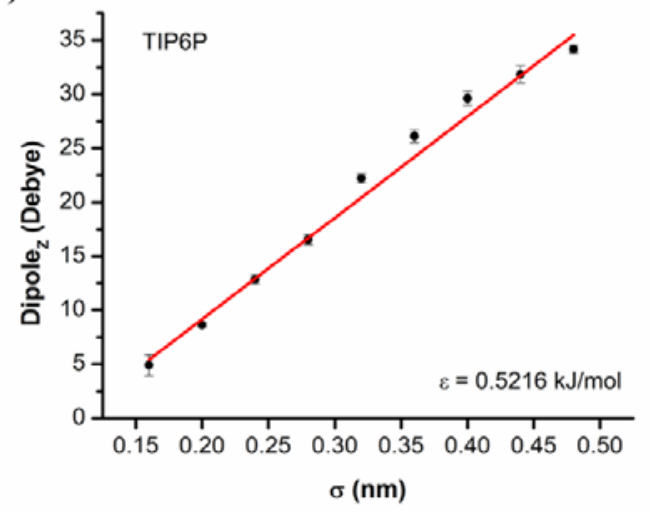

(c)

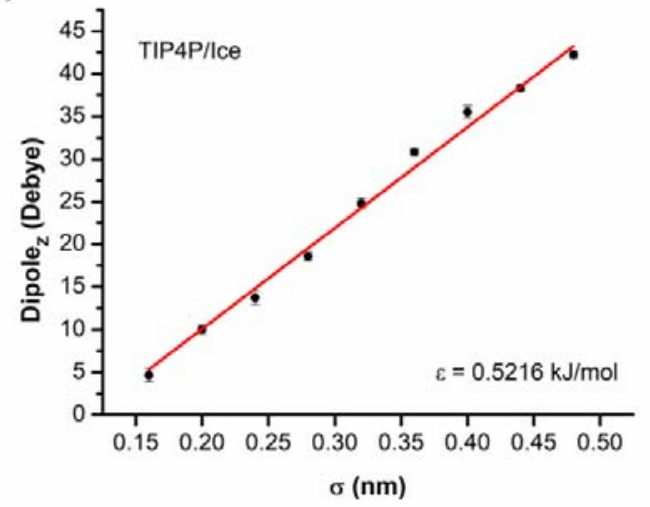

(b)

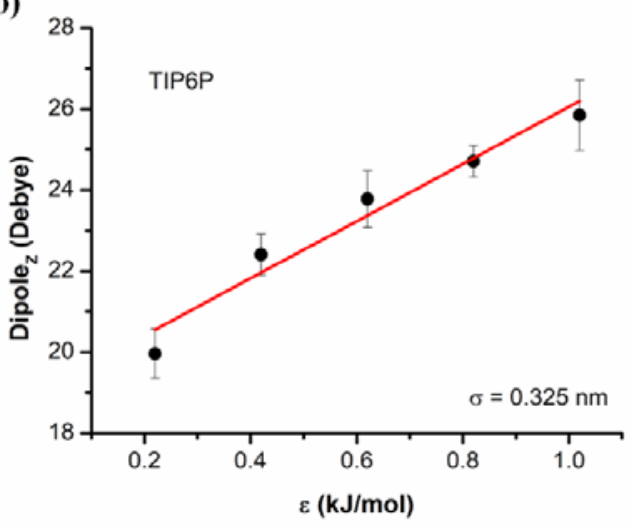

(d)

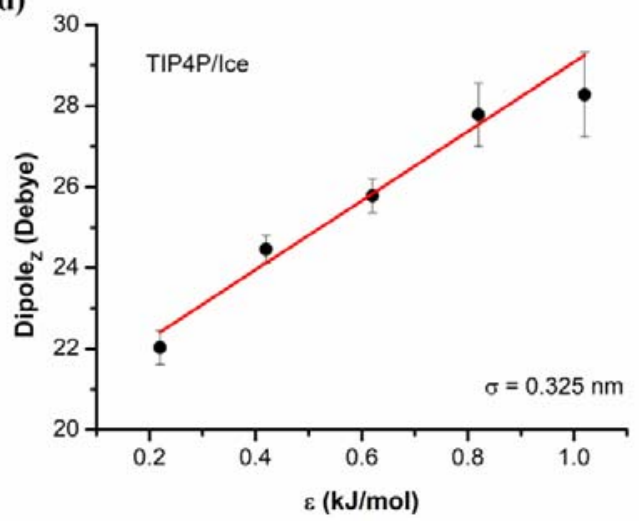

Figure S20. (a) The dipole projection along the z-direction as a function of the ionic size ( $\sigma$ is one of the parameters of the LJ potential, which corresponds to the ionic size) with the value of $\boldsymbol{\varepsilon}(\boldsymbol{\varepsilon}$ is one of the parameters of the LJ potential, controlling the interaction between the water and the ions on the mica surface) is constant with the TIP6P water potential. (b) The dipole projection along the z-direction as a function of $\boldsymbol{\varepsilon}$ with the value of $\boldsymbol{\sigma}$ is constant by using TIP6P water potential. (c) The dipole projection along the z-direction as a function of the ionic size with the value of $\varepsilon$ is constant by using TIP4P/Ice water potential. (d) The dipole projection along the zdirection as a function of $\varepsilon$ with the value of $\sigma$ is constant by using TIP4P/Ice water potential. Note: the red lines are the functions based on the fitting from the points in each figure. 

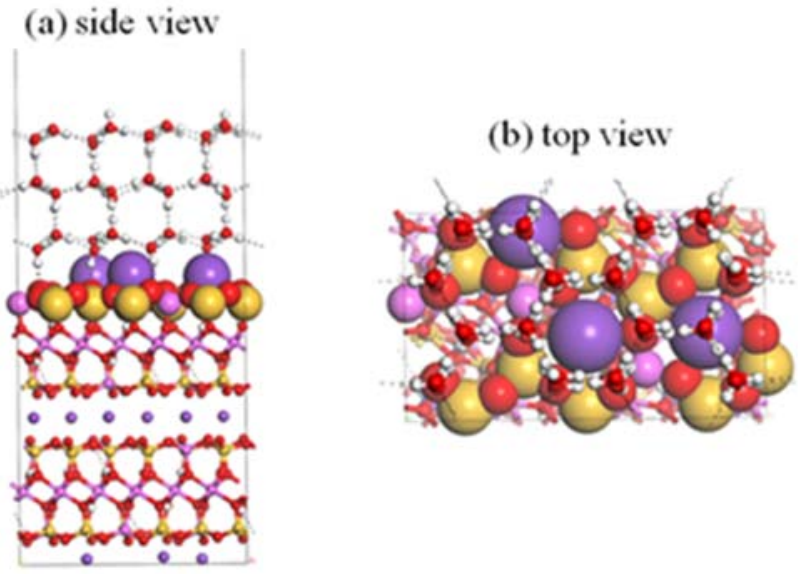

Figure S21. (a, b) The model of three-layer ice $\mathrm{I} h$ on top of mica used in the DFT computations. Color scheme: the yellow, red, white, pink, and light purple spheres represent the $\mathrm{Si}$, oxygen, hydrogen, $\mathrm{Al}$ atoms and $\mathrm{K}^{+}$of mica, and the black dashed lines represent hydrogen bonds. 


\section{REFERENCES}

(1) Li, K.; Xu, S.; Shi, W.; He, M.; Li, H.; Li, S.; Zhou, X.; Wang, J.; Song, Y. Investigating the effects of solid surfaces on ice nucleation. Langmuir 2012, 28, 10749-10754.

(2) Liu, K.; Wang, C.; Ma, J.; Shi, G.; Yao, X.; Fang, H.; Song, Y.; Wang, J. Janus effect of antifreeze proteins on ice nucleation. Proc. Natl. Acad. Sci. U. S. A. 2016, 113, 14739.

(3) Shen, Y. R. Surface properties probed by second-harmonic and sum-frequency generation. Nature 1989, 337, 519-525.

(4) Eisenthal, K. B. Liquid Interfaces Probed by Second-Harmonic and Sum-Frequency Spectroscopy. Chem. Rev. 1996, 96, 1343-1360.

(5) Lambert, A. G.; Davies, P. B.; Neivandt, D. J. Implementing the Theory of Sum Frequency Generation Vibrational Spectroscopy: A Tutorial Review. Appl. Spectrosc. Rev. 2005, 40, 103-145.

(6) Rey, R.; Møller, K. B.; Hynes, J. T. Hydrogen Bond Dynamics in Water and Ultrafast Infrared Spectroscopy. J. Phys. Chem. A 2002, 106, 11993-11996.

(7) Nada, H.; van der Eerden, J. P. J. M. An intermolecular potential model for the simulation of ice and water near the melting point: A six-site model of $\mathrm{H}_{2} \mathrm{O}$. J. Chem. Phys 2003, 118, 7401-7413.

(8) Abascal, J. L. F.; Fernández, R. G.; Vega, C.; Carignano, M. A. The melting temperature of the six-site potential model of water. J. Chem. Phys 2006, 125, 166101.

(9) Vrbka, L.; Jungwirth, P. Homogeneous Freezing of Water Starts in the Subsurface. J. Phys. Chem. В 2006, 110, 18126-18129.

(10) Vatamanu, J.; Kusalik, P. G. Observation of two-step nucleation in methane hydrates. Phys. Chem. Chem. Phys 2010, 12, 15065-15072.

(11) Zielke, S. A.; Bertram, A. K.; Patey, G. N. A Molecular Mechanism of Ice Nucleation on Model Agl Surfaces. J. Phys. Chem. B 2015, 119, 9049-9055.

(12) Zielke, S. A.; Bertram, A. K.; Patey, G. N. Simulations of Ice Nucleation by Kaolinite (001) with Rigid and Flexible Surfaces. J. Phys. Chem. B 2016, 120, 1726-1734.

(13) Zielke, S. A.; Bertram, A. K.; Patey, G. N. Simulations of Ice Nucleation by Model AgI Disks and Plates. J. Phys. Chem. B 2016, 120, 2291-2299.

(14) Cygan, R. T.; Liang, J. J.; Kalinichev, A. G. Molecular Models of Hydroxide, Oxyhydroxide, and Clay Phases and the Development of a General Force Field. J. Phys. Chem. B 2004, 108, 1255-1266.

(15) Perdew, J. P.; Burke, K.; Ernzerhof, M. Generalized Gradient Approximation Made Simple. Phys. Rev. Lett. 1996, 77, 3865-3868.

(16) Grimme, S.; Antony, J.; Ehrlich, S.; Krieg, H. A consistent and accurate ab initio parametrization of density functional dispersion correction (DFT-D) for the 94 elements H-Pu. J. Chem. Phys 2010, 132, 154104.

(17) Gaines; George, L.: The ion-exchange properties of muscovite mica. J. Phys. Chem. 1957, 61, 1408-1413.

(18) Xu, L.; Salmeron, M. An XPS and Scanning Polarization Force Microscopy Study of the Exchange and Mobility of Surface lons on Mica. Langmuir 1998, 14, 5841-5844.

(19) Stojilovic, N.: Why Can't We See Hydrogen in X-ray Photoelectron Spectroscopy? J. Chem. Edu. 2012, 89, 1331-1332.

(20) Odelius, M.; Bernasconi, M.; Parrinello, M.: Two-Dimensional Ice Adsorbed on Mica Surface. Phys. Rev. Lett. 1997, 78, 2855-2858.

(21) Atkinson, J.; Murray, B.; O'Sullivan, D.: Rate of Homogenous Nucleation of Ice in Supercooled Water. J. Phys. Chem. A 2016, 120, 6513-6520.

(22) Vali, G.: Interpretation of freezing nucleation experiments: singular and stochastic; sites and surfaces, Atmos. Chem. Phys. 2014, 14, 5271-5294.

(23) Tuladhar, A.; Chase, Z. A.; Baer, M. D.; Legg, B. A.; Tao, J.; Zhang, S.; Winkelman, A. D.; Wang, Z.; Mundy, C. J.; De Yoreo, J. J.; Wang, H.-f. Direct Observation of the Orientational Anisotropy of Buried Hydroxyl Groups inside Muscovite Mica. J. Am. Chem. Soc 2019, 141, 2135-2142. 